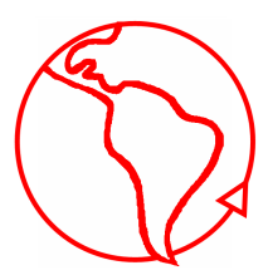

\title{
La prevención del consumo de drogas en el sistema educativo chileno: SENDA y la Escuela
}

\author{
Preventing Drug Abuse in the Chilean Educational System: SENDA \\ and School
}

Patricio Espinosa

Facultad de Ciencias Sociales, Universidad Católica de Temuco, Chile

\begin{abstract}
RESUMEN
El presente trabajo expone los resultados de una investigación respecto del quehacer preventivo en el sistema educativo del Servicio para la Prevención y Rehabilitación del Consumo de Drogas y Alcohol de Chile, SENDA. Situándonos desde una perspectiva de la teoría de sistemas sociales aplicada al análisis de sistemas organizacionales, caracterizamos su modelo de intervención analizando las lógicas que fundamentan su operar. Nuestra investigación concluye que, dadas las naturalezas programáticas tanto del SENDA como de las escuelas, la prevención del consumo de drogas se articularía a través de defraudaciones mutuas de expectativas, lo que invita a reflexionar acerca de las estrategias de intervención que plantea SENDA en el sistema educativo.
\end{abstract}

PALABRAS CLAVE: SENDA Chile; Sistemas Organizacionales; Prevención de Drogas; Modelo Dirigido; Conocimiento Situado

\section{ABSTRACT}

This paper presents the results of a research regarding the preventive task in the educational system of the Service for the Prevention and Rehabilitation of Drug and Alcohol of Chile, SENDA. From a perspective of social systems theory applied to the analysis of organizational systems, we characterize its intervention model analyzing the logics that underlie its operations. Our research concludes that, given the natures of both the SENDA program as schools, prevention of drug use would be articulated through mutual expectation disappointment, which invites us to think about intervention strategies posed SENDA in the educational system.

KEYWORDS: SENDA Chile; Organizational System; Drugs Prevention; Driven Model; Situated Knowledge

\section{INTRODUCCIÓN}

La prevención del consumo de drogas en el sistema educativo chileno posee una trayectoria que data desde la década de 1990, periodo en el cual se implementan iniciativas dependientes de los Ministerios de Educación y de Salud como los programas 'Quiero mi vida sin drogas' y los 'Proyectos de Prevención Escolar', PEP (Martínez \& Covarrubias 1999). En 2003, el Consejo Nacional para el Control de Estupefacientes,

REVISTA MAD - UNIVERSIDAD DE CHILE

Revista del Magíster en Análisis Sistémico Aplicado a la Sociedad

Facultad de Ciencias Sociales, Departamento de Antropología.

Universidad de Chile

www.revistamad.uchile.cl 
CONACE ${ }^{1}$ crea cuatro programas de carácter continuo y sistemático diseñados para ser implementados por educadoras de párvulo y profesores. Estos son los programas 'En busca del Tesoro' (dirigido a educación preescolar), 'Marori y Tutibú', 'Quiero Ser' (para educación básica) y 'Yo elijo' (para educación media). Con la creación en el año 2011 del Servicio Nacional de Prevención y Rehabilitación de Drogas y Alcohol, SENDA ${ }^{2}$, se incrementa la presencia de programas preventivos en el sistema educativo. Prueba de ello es la intensificación de la intervención preventiva en escuelas (programas 'Chile Previene en la Escuela', 'A tiempo' y 'Certificación de Escuelas Preventivas') y la implementación de un programa de prevención universal denominado 'Actitud', que reemplaza a los programas preventivos del nivel básico ('Marori \& Tutibú, 'Quiero Ser') y medio ('Yo Decido'). Todos estos programas (salvo 'A Tiempo') demandan, en distintos niveles de responsabilidad, el trabajo directo de los equipos docentes de escuelas y liceos.

Como vemos, estas estrategias preventivas han dado especial relevancia a la cobertura, lo que sin embargo contrasta con los resultados. En efecto, al observar la evolución de las prevalencias de consumo entre los años 2001 y 2011 (SENDA 2011) estas tienden a aumentar o a estabilizarse, pero salvo el tabaco, cuyas prevalencias día, mes y año tienden a descender, no se observan otros descensos estadísticos significativos (mayores a 0,5 puntos porcentuales)

La Estrategia Nacional de Drogas 2003 - 2008 (CONACE 2003: 26) planteaba el siguiente objetivo: "evitar y disminuir el consumo de drogas en niños, niñas y jóvenes de todas las instituciones educativas del país". Este enunciado contrasta con los indicadores para el período: la prevalencia anual de marihuana aumenta en 2,1 puntos porcentuales entre los años 2003 y 2009. Durante este mismo periodo, la cocaína y la pasta base aumentan levemente en 0,3 y 0,1 puntos porcentuales respectivamente. Los inhalables disminuyen en 0,2 puntos porcentuales y solo el alcohol experimenta un descenso significativo de 3,4 puntos porcentuales en la prevalencia anual.

Por su parte, la Estrategia Nacional de Drogas y Alcohol 2011-2014 (CONACE 2011) contempla como ejes prioritarios para la prevención de drogas en el sistema educativo la disminución en un 15\% de la prevalen-

\footnotetext{
${ }^{1}$ Dependiente del Ministerio del Interior, creado a través del Decreto Supremo no 683 del 21 de septiembre de 1990

${ }^{2}$ Es creado por medio de la Ley 20.502, siendo dependiente del Ministerio del Interior y Seguridad Pública de Chile.
} 
cia anual en los consumos de marihuana y alcohol, y la disminución de un $10 \%$ en la prevalencia de consumo anual de cocaína. Sin embargo, al revisar las prevalencias anuales de estas drogas para el periodo de los años 2009 y 2011 se observa que el consumo de marihuana experimenta un aumento en 4,4 puntos porcentuales. De igual manera, la prevalencia en el consumo anual de alcohol aumenta en 2,3 puntos porcentuales, mientras que la prevalencia anual de cocaína disminuye levemente en 0,2\% (SENDA 2011).

Estos antecedentes permiten evidenciar que la prevención del consumo de drogas en el sistema educativo no ha logrado los resultados deseados, lo que llama la atención si tomamos en cuenta la envergadura y continuidad programática del SENDA en el trabajo con escuelas. Esta es la relación que nos llevó a reflexionar en torno a las estrategias de intervención del SENDA en el sistema educativo.

Para ello, el presente texto inicia con la caracterización del modelo de intervención que construye SENDA para trabajar la prevención del consumo de drogas en el sistema educativo (sección 1). Luego, exponemos brevemente el enfoque epistemológico que guía las siguientes reflexiones (sección 2). Posteriormente, observamos a SENDA desde la teoría de sistemas sociales en el contexto de la diferenciación funcional (sección 3), para luego evidenciar las implicancias de su estrategia de prevención para el sistema educativo (sección 4). Finalmente, concluimos con una reflexión de lo aquí expuesto.

\section{LA ESTRATEGIA DEL SENDA: MODELO DIRIGIDO}

La complejidad es el problema básico de la sociedad contemporánea (Rodríguez \& Arnold 2007), lo que implica que cada uno de los problemas sociales, y su respectivo tratamiento a través de políticas públicas, no pueden ser observados de manera unitaria e integradora. Al contrario, cada observador los distinguirá de acuerdo a sus propios criterios de observación, por lo que una temática como la prevención del consumo de drogas en el sistema educativo generará múltiples y encontradas visiones. Con base en esto, proponemos la hipótesis señalando que, dadas sus naturalezas programáticas, el modelo de intervención de la organización preventiva (SENDA) es incompatible con la organización educativa (escuelas, liceos), por lo cual prima insatisfacción de expectativas entre ambas, y sobre todo, la comunicación por parte del SENDA de un concepto de prevención más cercano a un cumplimiento de metas autodefinidas que a un 
compromiso por desentrañar la complejidad de las relaciones al interior de cada comunidad educativa.

Montenegro (2001: 69) agrupa los diversos enfoques de intervención social en dos grandes modelos: "modelo dirigido" y "modelo participativo". A grandes rasgos, el modelo dirigido se fundamenta en un principio de responsabilidad (que puede ser, por ejemplo, estatal o de países ricos sobre países pobres), emergiendo desde un diagnóstico de desigualdad social, entendiendo que existen personas y colectivos con carencias. En este modelo encontramos principalmente los programas y servicios sociales del Estado y las ONG. Por otro lado, el modelo participativo, que surge a partir de las demandas de algún colectivo social, busca un sentido compartido de trabajo, en que la intervención se construya de manera dialógica entre sujetos de intervención e interventores, buscando instalar una relación igualitaria entre conocimiento experto y popular. En este modelo encontramos la educación popular, la teología de la liberación, la investigación acción-participativa y la psicología comunitaria, entre otros. Frente a la invisibilización del sujeto en el modelo dirigido, y a la legitimación ideológica en la lectura de lo social del modelo participativo -que terminaría por otorgar al conocimiento experto la atribución de aquello a intervenir-, Montenegro (2001: 235) propone la perspectiva de intervención "situada", en cuya base encontramos el concepto de contingencia ${ }^{3}$. La perspectiva situada posee, entre sus principales características, el concebir la intervención y al conocimiento desde diversas "posiciones de sujeto", siempre contingentes. Con "posiciones de sujeto", la autora se refiere a una suerte de encuentro dialógico entre posiciones a favor o en contra, imbuidas en relaciones de poder, entre personas afectadas, equipos interventores, asociaciones con intereses en juego, etc. que en conjunto se encaminen a poder definir aquello digno de intervenir; es decir, una propuesta que define la intervención desde la participación transversal de agentes de diversos ámbitos sociales. Así, el conocimiento ya no pertenecería solo a la ciencia, sino más bien a la articulación de significados "temporales y precarios" (Montenegro 2001: 308). La lógica tras el conocimiento situado sería que este se construye de manera contingente a partir de los diversos significados de sujetos en contextos específicos, y su objetivo sería el establecimiento de posiciones de diálogo para pensar los

\footnotetext{
${ }^{3}$ La contingencia es "todo lo que no es ni necesario ni imposible" (Luhmann 1997: 90), es decir, contingencia hace alusión a la exclusión de necesidad e imposibilidad, toda vez que se refiere a un dato respecto de alternativas posibles o a un dato que puede ser diferente de lo que es. Un dato es la selección de un ámbito de múltiples posibilidades.
} 
procesos de intervención, y no la búsqueda de respuestas finales a formas de intervención propiamente tales.

Buena parte de las estrategias del SENDA para el mundo educativo se sostienen en la psicología y sus variantes (social, clínica). Con base en ella se incorporan una serie de marcos teórico-conceptuales que fundamentan las diversas acciones tendientes a prevenir el consumo de drogas al interior de escuelas y liceos. Dentro de los principales encontramos el enfoque de "factores protectores y de riesgo de consumo de drogas" y al "modelo de desarrollo de competencias". El primero se vincula a identificar y trabajar en aquellas situaciones o elementos que bien probabilizan o improbabilizan un consumo de drogas (SENDA 2012; CONACE 2005, 2011, 2008), mientras que el segundo busca facilitar condiciones que permitan el desarrollo de fortalezas psicológicas y comunitarias para generar autonomía en la resolución de conflictos (Arón 1992 en CONACE 2005). En esta misma línea encontramos la perspectiva "ecológica", la cual vincula el desarrollo personal a las condiciones de diversos dominios ambientales (Gifre \& Moisés 2012). Otros enfoques son la teoría del "apego" (Bowlby 1990 en CONACE 2005), el enfoque de "resiliencia" (Ruter 1993 en CONACE 2005) y el enfoque de "derechos infanto-adolescentes" (UNICEF 1989)

Estos son los principales enfoques teóricos que sustentan la comprensión del sujeto de intervención y el problema a tratar, esto es, los supuestos epistemológicos y ontológicos que orientan el quehacer de SENDA en el sistema educativo. Acá, por ejemplo, es posible identificar que cierto segmento de su población objetivo -adolescentes- presenta grandes desafíos para el trabajo preventivo debido a su edad y sus consecuentes características psicológicas y emocionales: "La etapa de la adolescencia es altamente vulnerable al consumo y abuso de drogas. En esta etapa de la vida se generan una serie de tensiones y necesidades" (CONACE 2007: 87). Además, la escuela aparece como el mejor de los contextos para identificar conductas de riesgo, prevenir consumos o bien para poner atajo a incipientes consumos de drogas: "Los diferentes estudios realizados en el mundo plantean que una de las alternativas de solución más importantes para abordar efectivamente el problema del consumo de drogas es a través de una educación preventiva en la escuela" (CONACE 2007: 07), "el trabajo preventivo (...) implica conocer y apoyarse en las políticas sociales de manera integrada, e implementar acciones focalizadas en fenómenos específicos que favorezcan la integración social" (SENDA 2012: 48). Entonces, tenemos un segmento social que estaría más afecto a iniciar un 
consumo problemático de drogas y un contexto de intervención con características únicas para el despliegue de estrategias de prevención, las que además permitirían reducir brechas de desintegración social en los educandos. De esta manera, SENDA construiría el problema del consumo de drogas justamente desde la óptica de la desintegración social concibiendo a los educandos como sujetos susceptibles a desviarse de las normas y patrones conductuales deseables para la sociedad. Esta perspectiva estaría muy cercana a las premisas de la teoría de la "anomia", en su versión mertoniana (López 2009), la cual señala que las conductas divergentes son fruto del desajuste entre los fines ideales de la sociedad y los medios para alcanzarlos. Así, la prevención del consumo de drogas se posicionaría en el sistema educativo como un recurso o medio que contribuye a los ideales valórico-normativos de la sociedad.

De ello se desprende que el problema del consumo de drogas en la población escolar radicaría en un fallo de las reglas de convivencia social, lo que llevaría al estudiante a 'distorsionar' su toma de decisiones, motivo por el cual SENDA se posicionaría para intervenir con el objetivo de buscar equilibrios sociales. En consecuencia, los estudiantes aparecen como un colectivo que bien se encuentra excluido o en riesgo de exclusión de recursos de diversos órdenes (económicos, sociales, culturales). Estas serían características de un modelo de intervención dirigido por cuanto se plantea la necesidad de intervenir desde el principio de la responsabilidad del Estado en un problema social que escinde a sujetos de los recursos que les llevarían a una vida sin drogas. SENDA, al intervenir en el sistema educativo, buscaría la integración de grupos sociales con carencias, siendo los adolescentes sus principales referentes toda vez que estos no poseerían información preventiva y serían más susceptibles a iniciar un consumo de drogas que otros grupos etarios.

En una primera síntesis: el problema de drogas en el sistema educativo se construye desde las altas expectativas hacia la escuela como contexto ideal para la prevención del consumo de drogas, los altos indicadores de consumo, y desde la aplicación del modelo de intervención que observa el consumo de drogas como una conducta desintegrada, y al grupo objetivo (escolares) como un colectivo social en desventaja (CONACE 2007). Estos puntos de análisis pueden darnos luces acerca de la experiencia del modelo de intervención del SENDA en el sistema escolar. Se trata de un modelo que podemos caracterizar, siguiendo a Montenegro (2001: 165) como modelo "dirigido", para el cual "el cambio social es posible siempre que sea a pequeña escala y funcional para la sociedad y siempre 
que esté avalado por políticas definidas desde quienes financian la intervención y el saber técnico de quien la lleva a cabo".

\section{PERSPECTIVA DE ANÁLISIS: LA COMPLEJIDAD Y LAS MÚLTIPLES POSIBILIDADES DE OBSERVACIÓN.}

Para Luhmann (2007) la comunicación es la única operación estrictamente social, la cual es llevada a cabo de manera autorreferencial por tres tipos de sistemas sociales: interacción, organización y sociedad. La interacción utiliza la co-presencia de personas como criterio de delimitación comunicando temas, la organización se delimita en torno a una membresía comunicando decisiones mientras que la sociedad es el sistema que comprende todas las comunicaciones. La sociedad moderna -o contemporánea-, a diferencia de sus predecesoras ${ }^{4}$, es un tipo de sociedad que se organiza en torno a la diferenciación funcional, lo que se traduce en que atiende a sus problemáticas a través de diversos sistemas parciales que se erigen en torno a funciones específicas, como el sistema educativo, el sistema político, el científico o el económico entre otros. Así, dependiendo del sistema observador (pensemos en organizaciones, por ejemplo), las posibilidades de describir un fenómeno social son múltiples.

Es así que para analizar el problema que aquí desarrollamos nos situamos desde el "programa sociopoiético" (Arnold 2005) al asumir la existencia de varios niveles de observación en torno a una temática de interés, para nuestro caso, la relación de la prevención del consumo de drogas con el sistema educativo: "La fortaleza de la perspectiva sociopoiética se extrae de premisas que apoyan aproximaciones metodológicas para tratar problemas específicos, y que permiten considerar las distintas racionalidades que lo constituyen" (Arnold 2005: 323). Varios planos de observación, ciertamente, dan cuenta de la complejidad que caracteriza a la sociedad contemporánea, la cual constantemente nos plantea el desafío de asirla a la luz de las ciencias sociales.

El rendimiento del programa sociopoiético se inscribe en la complejidad de la sociedad contemporánea, la cual se expresa en su policontextu-

\footnotetext{
${ }^{4}$ Para Luhmann (2007), han existido cuatro tipos de sociedades: la sociedad arcaica, caracterizada por la igualdad de los sistemas parciales (pensemos en bandas cazadoras recolectoras que definen funciones por criterios de interacción cara a cara y pertenencia a núcleos familiares); la sociedad centro-periferia, que plantea la diferencia desde la noción de un centro político administrativo, léase entre ciudad/campo o civilizado/bárbaro (Egipto, Mesopotamia, etc.); la sociedad estratificada, basada en la formación de la nobleza (propia de Europa de la Edad Media tardía hasta el siglo XVII), y por último, la sociedad moderna (actualidad), donde prima la diferenciación por funciones y se caracteriza por ser policéntrica.
} 
ralidad, es decir, en sus múltiples posibilidades para describirse. Si atendemos al problema del consumo de drogas, SENDA representaría una de las múltiples observaciones hacia esta temática, la cual, dado su grado de especialización, influiría y determinaría otras miradas sobre el mismo fenómeno. Frente a esta lectura de la sociedad, el programa sociopoiético operacionalizado a través de la observación de segundo orden ${ }^{5}$ - nos permitiría comprender el cómo SENDA distingue a las comunidades escolares, y desde allí, reflexionar en torno a la naturaleza misma del trabajo preventivo al interior de las escuelas y liceos.

\section{SENDA COMO SISTEMA ORGANIZACIONAL}

Como hemos señalado antes, los sistemas organizacionales corresponden a un tipo de sistema social autopoiético, clausurado operativamente, cuya característica principal es enlazar comunicaciones en forma de decisiones. Siguiendo a Andersen (2003b), los sistemas organizacionales están clausurados a la participación (membresía), pero abiertos a los programas y códigos de varios sistemas funcionales. Cabe señalar que este tipo de sistema social es propio de la modernidad, y su generalización ha sido tal que hoy se constituye como el "componente central del paisaje contemporáneo" (Arnold 2008: 90)

Al observar al SENDA como sistema organizacional, vemos que es capaz de asociarse a diversos sistemas funcionales tomando para sí sus códigos y programas. Aquí encontramos al sistema médico (salud/enfermedad), al sistema científico (verdadero/falso), al educativo (mejor/peor) y al político (gobierno/oposición) principalmente. Sus operaciones recursivas se efectúan al alero de estos sistemas funcionales, y al convertir toda comunicación en decisiones (la principal característica de los sistemas organizacionales), SENDA, paradójicamente, incrementa la complejidad al auto-obligarse a generar nuevas selecciones mediante nuevas decisiones. Es un lenguaje que se hace hacia delante (Arnold 2008) toda vez que los sistemas organizacionales son verdaderas "máquinas de decisión" (Nassehi 2005).

Como sistema organizacional, el SENDA es además un sistema constitutivo de sentido. El sentido es "la premisa para la elaboración de

\footnotetext{
${ }^{5}$ Desde una perspectiva luhmanniana, la observación de segundo orden se refiere a la observación de observaciones y observadores, asumiendo a la comunicación como el único elemento estrictamente social; es decir, la observación de segundo orden se plantea como un recurso teórico-metodológico para la construcción de conocimiento.
} 
toda experiencia" (Corsi et al. 1996: 64); es el médium por medio del cual los sistemas sociales pueden controlar el acceso a las múltiples posibilidades comunicativas en el marco de la complejidad que -como hemos señalado- caracteriza a la sociedad contemporánea. De esta manera, las organizaciones son capaces de comunicar/distinguir entre las tres dimensiones en las que justamente se articula el sentido, estas son: dimensión objetual, social y temporal. Cada una de estas dimensiones se articula de acuerdo a distinciones duales. Para la dimensión objetual es determinado/indeterminado, para la dimensión social es alter/ego y para la dimensión temporal antes/después, las que en su conjunto permiten -al ser el fundamento basal de la selectividad- distinguir $\mathrm{u}$ "ordenar la complejidad" (Izuzquiza 2008), y a su vez, distinguir tres niveles de experiencia diferenciados que forman la unidad del sentido, puesto que solo a través suyo es posible hablar de objetos, de tiempo y comunicación.

Las decisiones comunican expectativas sociales articuladas en estas tres dimensiones de sentido. Para ello, separan al mundo entre un antes y un después, por lo cual, la decisión permite a la organización diferenciar un ámbito de contingencia en el presente (la forma de la decisión), y de ahí, las decisiones van creando nuevas expectativas sociales vinculadas a decisiones posteriores. Pero esta comunicación de expectativas no significa satisfacción de las mismas, por cuanto surgirán nuevas decisiones de mayor y menor alcance tendientes a vincularse entre sí para determinar relevancias e interdependencias (Arnold 2008: 96). El antes en la forma de la decisión define contingencia abierta, el punto en que gran parte todavía puede ser cambiado ante la latencia de diversas posibilidades. El después, en la forma, plantea contingencia fija en relación a posibilidad de haber alcanzado una decisión diferente (Andersen 2003a). Cabe mencionar que las posibilidades de decisión actúan a través de "premisas decisionales" (Martens 2006: 90), las que se presentan en tres formas: a través de programas que evalúan las decisiones, en vías de comunicación para restringir las posibilidades de decisión (la más común es la estructuración jerárquica) y en la cualidad de la membresía (carrera, conocimientos, experiencias, prestigio, etc.). Estas premisas decisionales constituyen estructuras de expectativas al interior de la organización, y finalmente son los elementos que le otorgan operabilidad.

Vemos, pues, que el sistema organizacional SENDA incrementa complejidad por medio de sus decisiones, las que continuamente generan nuevas decisiones orientadas a comunicar/decidir sobre otras. Como señala Arnold (2008: 96), "decidir implica reconocer alternativas, para luego 
calcular sus consecuencias y finalmente ingresarlas en sus operaciones". Así, la decisión es un mecanismo que permite a la organización tratar con los riesgos e incertidumbres autoformulados, siendo su función, justamente, la reducción de niveles de riesgo e incertidumbre organizacionales.

Por ende, SENDA como sistema operativamente clausurado, enlaza comunicaciones en forma de decisiones permitiéndole definirse a sí mismo como organización especialista en el tratamiento de la prevención del consumo de drogas. Las implicancias de concebirlo como sistema social nos llevan a la pregunta por la construcción del problema de drogas, esto es, el entorno de SENDA.

Todo sistema se corresponde con un determinado entorno: "No se da la constitución de un sistema sin una relación con el entorno, y tampoco un entorno sin sistema: surgen solamente juntos" (Corsi et al. 1996: 149). Al operar por medio de sus propias reglas, el sistema organizacional SENDA construye su entorno de acuerdo a sus propias lógicas de observación/distinción, por ende, relevando y opacando dimensiones de sentido. SENDA construye el problema del consumo de drogas en escolares desde su propia autorreferencialidad, en función de sus propias determinaciones, pues, independiente de que se pueda argumentar la preexistencia de un entorno (problema del consumo de drogas), su relevancia surge desde su comunicación. Podemos de esta manera aproximarnos para comprender el cómo se va perpetuando un estilo preventivo con la misma lógica de intervención en escuelas, esto es, sin modificaciones sustanciales independiente de los resultados que arrojan los Estudios en Población Escolar (SENDA 2011). Siguiendo a Arnold (2008: 98), "las organizaciones hacen lo que hacen", pues se concentran en su propia viabilidad centrándose, exclusivamente, en su propósito originario.

En síntesis, SENDA, como sistema organizacional comunica decisiones, y se van configurando a sí mismas a través de la actualización de otras decisiones. Con esta misma operación recursiva construye autorreferencialmente su entorno, comunicando expectativas sociales de decisiones posteriores. En este andar decisional incrementan complejidad, pero a su vez reducen niveles de incertidumbre.

Dicho lo anterior, nos interesa observar al sistema organizacional SENDA en su relación con otros sistemas, específicamente como ellas comunican la intervención para el sistema educativo. 


\section{ORGANIZACIÓN PREVENTIVA/ORGANIZACIÓN EDUCATIVA}

Para el SENDA, la escuela es el espacio perfecto para llevar a cabo acciones preventivas del consumo de drogas, pues sus estudios señalan que en edades tempranas (escolares) es cuando se inicia el consumo:

Existe evidencia científica que muestra que el período en donde se concentra el mayor riesgo de iniciar consumo o desarrollar dependencia a sustancias adictivas es hasta los 21 años. Después de esta edad, las probabilidades de desarrollar un consumo problemático casi desaparecen. Los establecimientos escolares constituyen un espacio ideal para detectar precozmente posibles factores de riesgo entre los adolescentes y jóvenes. Los profesores, así como directores, sostenedores y apoderados, entre otros actores de la comunidad educativa, son agentes preventivos debido a su cercanía con los alumnos, a su papel como modelos y a su función educadora. Es en los colegios donde es posible llegar a los jóvenes escolarizados en la edad de máximo riesgo de inicio de consumo de drogas y alcohol, facilitándose así realizar programas de prevención. (SENDA s.f.)

Se trata de un contexto que mantiene fidelizado -la mayor parte del día- a niños y adolescentes, lo cual permitiría prever actitudes y patrones de conducta 'indeseables'. Se comunica el problema del consumo de drogas poniendo énfasis en la necesidad de intervenir al interior de la escuela, generándose así expectativas de rol con los educandos (sujetos de intervención), con docentes y otros miembros de la comunidad educativa. Para el SENDA, la escuela reviste un contexto especialmente sensible al consumo de drogas, siendo sus profesores distinguidos con altas expectativas de rol preventivo.

Por otro lado, las escuelas operan mediante programas específicos que les permiten buscar cambios planificados en el educando. Hablamos de planes de estudio, los que se construyen desde el nivel ministerial. Por encima de las calificaciones por asignaturas, que permiten medir el progreso en el conocimiento de cada educando, existe una evaluación que ciertamente se orienta a medir los progresos de la organización escolar. Nos referimos al Sistema de Medición de Resultados de Aprendizaje, la prueba SIMCE, mecanismo destinado a evaluar los resultados de aprendizajes de los establecimientos y que es construido sobre la base de competencias esperables por nivel educativo. Este mecanismo de evaluación se centra en la medición de solo una parte del currículum educativo y en algunos niveles de la escolaridad, excluyendo a los contenidos valóricos que se desprenden de los Objetivos Fundamentales Transversales de la Educa- 
ción Básica y Media (OFT), en los que, justamente, se sitúa la prevención del consumo de drogas. Esto implica que los establecimientos educacionales concentren la mayor parte de sus energías en los contenidos susceptibles de evaluación, y en consecuencia, empobreciendo el currículum educativo. También se tiende a la desprofesionalización del rol docente al perderse su injerencia sobre la semántica de la calidad educativa, quienes, al estar constantemente presionados a cumplir con resultados, inevitablemente verán afectada su salud laboral, repercutiendo en la salud emocional de los estudiantes. Esta misma presión es la que invita a los establecimientos educacionales a reducir las horas destinadas a aquellos contenidos que no se evalúan, volcando al mundo educativo a una lógica de competencia, y no tanto de cooperación (OPECH 2005, Redondo 2005). En los establecimientos educacionales las decisiones más relevantes apuntarían, en gran medida, a un buen rendimiento en esta prueba. No es de extrañar que al interior de escuelas y liceos las decisiones en el campo de la prevención estén siempre sujetas a la energía puesta en sus quehaceres programáticos (objetivos de aprendizaje), relegando la prevención del consumo de drogas a la obtención de materiales preventivos, a algunas acciones específicas y a la visita esporádica de agentes preventivos.

De los programas que el SENDA dispone para el mundo educativo, 'Actitud' (Activa tu desarrollo) es el único de carácter continuo, estando presente en todos los niveles de la educación desde $1^{\circ}$ básico hasta $4^{\circ}$ medio. Este programa está diseñado con objetivos, contenidos y un número determinado de sesiones ${ }^{6}$ medidas en horas pedagógicas para ser aplicado a estudiantes al interior de aula por parte de los profesores. 'Actitud' es incorporado en los establecimientos educacionales siempre que estos lo soliciten, contemplando la participación activa de profesores en la aplicación de sus sesiones de trabajo, cada una de las cuales tiene una duración de 60 minutos. Pero son conocidas las dificultades que los docentes deben hacer frente en el campo educativo, sobretodo en el sistema público y subvencionado, las que van desde la alta concentración de estudiantes por aula, pasando por las escasas horas docentes a tareas educativas más allá de las clases en sala (Redondo 2009), hasta la naturaleza misma de la gestión administrativa de los establecimientos educacionales, cuyo excesivo centralismo cristaliza en la aplicación de programas de estudio altamente formales al interior del aula, lo que genera distancias entre las premisas de los instrumentos normativo-programáticos de las escuelas y lo que ocurre al interior de la sala (Silva 2009). Si nos centramos en las competencias

\footnotetext{
${ }^{6}$ Seis para estudiantes, dos para los apoderados (SENDA, s.f. b; s.f. c)
} 
docentes en el campo preventivo del consumo de drogas, es muy probable que la gran mayoría no posea competencias en el área específica de la prevención (biología, química, psicología, drogas, etc.). Desde aquí sería posible observar una distorsión en las expectativas de rol con las que SENDA distingue al profesorado, pues se le pide realizar una tarea para lo cual no estaría capacitado, para la que no tendría tiempo y más aún, que no forma parte de sus prioridades programáticas. De este modo, las metas y acciones preventivas de SENDA se articularían en función de criterios que, más bien, desconocerían posibilidades de acción docente, tiempos escolares y acceso relevante a las familias.

SENDA plantea como principal objetivo la búsqueda de un trabajo preventivo autónomo por parte de las comunidades escolares (SENDA 2012; CONACE 2007) a través del despliegue de sub-programas y acciones asociadas. De hecho, incorpora la necesidad de inclusión curricular de la prevención como un giro que busca validación y fundamento en las propias lógicas educativas:

Entendemos por inclusión curricular el proceso de instalación de temáticas preventivas en los distintos espacios pedagógicos (contenidos mínimos obligatorios, objetivos fundamentales transversales, reuniones de apoderados, consejos de curso, entre otros), de manera que se garantice que tanto los niños, niñas y adolescentes del establecimiento escolar, así como sus familias, sean sujetos de prevención. (CONACE 2011: 36).

Sin embargo, la mayor parte de estas acciones terminarían por descansar casi exclusivamente en el trabajo docente, pues, en la práctica, llevarlas a cabo implicaría una ruptura en los tiempos destinados a la enseñanza de contenidos instrumentales y una sobrecarga laboral para los docentes. Estos sub-programas y acciones, si bien se validan en cada establecimiento educacional (toda vez que los equipos directivos de escuelas y liceos confirman las acciones de un determinado plan de trabajo preventivo presentado por SENDA), no lograrían incorporarse a las prácticas pedagógicas, pues las escuelas los observarían como programas externos y altamente especializados. Para la escuela, las acciones y sub-programas de SENDA tendientes a la prevención de drogas en el sistema escolar serían, justamente, metas de SENDA. Esto se debe a la naturaleza del modelo de intervención de SENDA, (modelo dirigido) el cual define una situación problemática susceptible de ser conocida e intervenida a través de políticas basadas, más bien, en la experticia (Pinilla 2012), esto es, a través de particulares formas de saber que articulan el conocimiento científico con la 
política pública, modelando la toma de decisiones políticas a través de la discriminación de información que permite finalmente concebir la prevención para el sistema educativo. En otros términos, políticas que deciden cómo será la prevención de drogas en escuelas, la respuesta de estas a las diversas acciones preventivas y los resultados esperados.

En consecuencia, para el SENDA, la formulación del problema del consumo de drogas en escolares -que otorga sentido a su modelo de intervención- existiría con independencia de la población objetivo, lo que implicaría la aplicación per se de sus diversas estrategias. Hablamos de un concepto de realidad que antecede al sujeto de intervención y de acciones universales que persiguen la constitución de una cultura preventiva en escuelas y liceos (programa 'Actitud', estrategias 'habilidades para la vida', programa de 'certificación de escuelas', realización de 'ferias preventivas', etc.)

No obstante, los programas 'A Tiempo' y 'Chile previene en la escuela' plantean un modelo distinto. El primero realiza atención dirigida a estudiantes de $7^{\circ}$ básico a $2^{\circ}$ medio que presentan mayor riesgo de consumo, implementando para ello acciones preventivas bajo la forma de consejerías individuales, asesorías familiares y trabajo con la red social comunal a través de seis sesiones individuales, seis grupales y tres con sus familias (SENDA 2012). Por otro lado, 'Chile previene en la escuela' dinamiza una serie de acciones a cargo de un gestor (funcionario SENDA). Estas se orientan a: articular la oferta de prevención, evaluar la efectividad de los programas preventivos ya existentes y promover estrategias de prevención. Tanto 'Chile previene en la escuela' como 'A Tiempo' plantean la novedad de la inserción de profesionales al interior de la escuela, quienes se ocupan en diagnosticar y trabajar en torno a factores de riesgo y protección del consumo de drogas en dimensiones individuales y comunitarias. Sin embargo, solo trabajan con aquellos establecimientos educacionales que presentan un índice de vulnerabilidad escolar superior a 70 puntos. Además, debido a su costo, son programas con los que cuentan solo algunas comunas, sin mencionar que, por la exigencia de las funciones, cada funcionario puede hacerse cargo de un número reducido de establecimientos educacionales. En consecuencia, ambos programas plantean un giro en la intervención, donde la apuesta estaría en ganar más en profundidad que en cobertura. Tendremos que esperar los resultados de los próximos estudios en población escolar para observar los efectos de la instalación de estos programas al interior de los establecimientos educacionales. No obstante, nos inclinamos a pensar que esta lógica se acercaría más a 
conseguir los resultados esperados, pero aun así, al estar delimitada al cumplimiento de metas organizacionales (metas de SENDA), pueden generarse efectos no deseados, como apatía docente hacia las acciones preventivas frente a la presencia de profesionales que, perteneciendo a SENDA, trabajan cada uno por separado en una misma escuela y enfocados en sus respectivas metas. Se puede ganar mucho en visibilización programática, erigir al establecimiento como uno que se preocupa de la prevención, pero desconocemos cuanto se pueda ganar en prevención de drogas toda vez que esta se mide por casos atendidos ('A tiempo') o por la instalación y desarrollo de un número determinado de fases programáticas ('Chile previene en la escuela').

Para finalizar, sostenemos que en la prevención del consumo de drogas hacia el sistema educativo obviamente el docente tiene muchísimo que aportar en la construcción de una intervención más reflexiva y comprensiva con los roles de cada uno de sus participantes (interventores e intervenidos). Incluso asistentes, inspectores, auxiliares, vendedores establecidos, transportistas y demás funcionarios de la escuela también debiesen ser incluidos en este modelo de intervención, pues muchas veces son ellos quienes logran generar vínculos emocionales y complicidad con los estudiantes en sus horas de recreo y esparcimiento. Si pensamos desde una perspectiva de "conocimiento situado" (Montenegro 2001: 264) estos sujetos serían totalmente pertinentes e imprescindibles para definir la estrategia preventiva en la escuela, pues sus significados -contingentemente constituidos- aportarían a establecer acciones validadas por el conjunto de la comunidad escolar -y ya no solo desde el conocimiento experto-, y así, el producto final sería una estrategia preventiva de la cual son responsables sujetos con variados roles al interior de la escuela. En el fondo, la apuesta del "conocimiento situado" se encuentra en incorporar el problema de la contingencia en la intervención, y sobre todo, en la inclusión de múltiples miradas en torno a aquello que se intervendrá, invitándonos a pensar el conocimiento ya no solo desde la experticia, y en una realidad que no existe de forma independiente del observador.

\section{COMENTARIOS FINALES}

De manera sintética, podemos resumir los resultados antes expuestos de la siguiente manera: 
SENDA, como sistema organizacional especializado, comunica la prevención del consumo de drogas con un programa particular de intervención dirigido al sistema educativo.

Este programa -como ha sido la tónica desde 2003- distingue al profesorado como los principales agentes preventivos al interior de los establecimientos.

Ante lo cual, se generan altas expectativas de rol preventivo, donde se precisa que deban aplicar programas preventivos de alcance anual, abrir espacios de conversación adolescente-juvenil y abordar temáticas sensibles con los educando (vinculadas con el consumo de drogas)

Por su parte, las organizaciones educativas (escuelas) participan de la comunicación preventiva, lo que inferimos toda vez que estas voluntariamente solicitan materiales y apoyo preventivo a SENDA (se 'entiende' la importancia del mensaje preventivo para el mundo educativo)

Sin embargo, para las escuelas, la prevención de drogas sería extraescolar por cuanto se comunica a partir del campo reflexivo de una organización técnica y altamente especializada. Por ende, la expectativa de las organizaciones escolares estaría más cercana a la idea de atención especializada para sus educandos.

Sumado a lo anterior, las escuelas considerarían en esta distinción la carga laboral, las exigencias del Ministerio de Educación en el área específica de la evaluación organizacional (SIMCE), la escasa especialización en el campo preventivo por parte de los profesores, además de los casi nulos espacios para aplicar programas extraescolares que, como 'Actitud', exigen lógicas de aprendizaje estandarizadas (sesiones con objetivo, minutos de duración, contenidos especializados, etc.)

Algunos resultados serán la escasa aplicación de materiales preventivos por parte de los profesores, además de escasos espacios de reflexión en torno a estas temáticas. Sin embargo, las oficinas comunales de SENDA cumplirán sus metas de trabajo autoimpuestas, confirmando su viabilidad institucional en el campo de la prevención de drogas.

Por su parte, las escuelas seguirán enfocadas en sus propias metas de aprendizaje habiendo esperado al final del día una intervención 'más presente' por parte de SENDA. Se apelará a los altos rendimientos que otorgaría la presencia de 'externos y especialistas' para trabajar temas preventivos al interior del aula de clases.

Hemos intentado caracterizar el modelo de intervención de SENDA, el organismo encargado de la prevención del consumo de drogas en Chile, y desde ahí ponerlo en relación con el sistema educativo desde una 
particular lectura teórica. Hemos visto que SENDA incorpora en su discurso diversos conceptos vinculados a un tratamiento participativo de la problemática del consumo de drogas en población escolar. Se habla, por ejemplo, de la necesidad de incorporar a la familia y del involucramiento pleno de los docentes. Pero en la práctica, las condiciones ideales no se darían en los contextos educativos, y así, la prevención de drogas para el sistema escolar termina siendo en algunos casos un mero trabajo individual, no adaptado, incompleto y acusando agotamiento de procesos en estudiantes y docentes. La intervención se remite a la aplicación de programas estructurados desde el nivel central (Santiago) y poco adaptables a realidades locales.

El modelo de intervención que caracteriza a SENDA nos invita a pensar en que una temática tan compleja como la prevención del consumo de drogas en el sistema educativo es necesario articularla desde una multiplicidad de actores, lo que atañe a la posibilidad de re-pensar en torno a quienes serán concebidos como agentes preventivos más allá del cuerpo docente, lo que en consecuencia permita visibilizar y poner en diálogo a diversas posiciones de sujeto.

Observar la relación entre SENDA y el sistema escolar desde una perspectiva "situada" (Montenegro 2001) implica el desarrollo de interrogantes de orden ontológico y epistemológico principalmente, las que nos permiten problematizar las categorías centrales con las que se comprende el fenómeno (intervención, prevención, trabajo en aula, comunidades educativas). También nos permite analizar las expectativas de rol que emanan por entre los sujetos involucrados en la intervención. Por último, nos invita a reflexionar en las formas de conocimiento que subyacen a la intervención preventiva, y desde allí, a re-pensar en torno al involucramiento de los agentes de diferentes ámbitos en la definición del problema social que se desea abordar.

Brindar una propuesta de intervención preventiva del consumo de drogas para el sistema escolar excede el alcance del presente artículo. No obstante, sostenemos que una estrategia de intervención tendiente a prevenir el consumo de drogas al interior de escuelas y liceos debiese considerar a la totalidad de actores de la comunidad escolar. Si bien el SENDA incorpora el concepto de comunidades escolares, este se desglosa más bien en roles docentes asociados a cumplimientos de metas (miembros del equipos directivo, jefe de unidad técnico pedagógica, docentes de aula, inspectores, familias), invisibilizando a otros actores que, como señalábamos, pueden generar iguales o mayores niveles de amistad y complicidad 
con los educando (auxiliares, personal de cocina, asistentes, vendedores establecidos, etc.). Además, llama la atención que frente a la relevancia que se otorga a la familia en la prevención del consumo de drogas en estudiantes (CONACE 2011; SENDA 2012) las sesiones de trabajo contempladas sean tan escasas (para 'Actitud' son 2 sesiones, 'A Tiempo' contempla 3 sesiones). Finalmente, solo nos resta reforzar las bondades de plantear la prevención de drogas desde una lógica de inclusión profesional al interior de las escuelas, acción mediante la cual se podría contribuir a aminorar la defraudación de expectativas de las escuelas hacia la prevención, facilitando la articulación de los diversos actores sociales de la comunidad escolar en función de generar las bases de una cultura preventiva del consumo de drogas. RM

\section{BIBLIOGRAFÍA}

Arnold, M. (2003). Fundamentos del constructivismo sociopoiético. Cinta de Moebio, $18,162-173$.

Arnold, M. (2005). La sociedad como sistema autopoiético: Fundamentos del programa sociopoiético. En F. Osorio, M. Arnold, S. González \& E. Aguado (Eds.), La nueva teoría social en Hispanoamérica. Introducción a la teoría de sistemas constructivista (pp. 46-71). México D.F.: Universidad Autónoma del Estado de México.

Arnold, M. (2008). Las organizaciones desde la teoría de los sistemas sociopoiéticos. Cinta de Moebio, 32, 90-108.

Andersen N. A. (2003a). The Undecidability of Decision. Autopoietic Organization Theory. Working Paper No. 12. Copenhagen. Copenhagen Bussines School.

Andersen N. A. (2003b). Polyphonic Organisations. En T. Bakken \& T. Hernes (Eds.), Autopoietic Organization Theory: Drawing on Niklas Luhmann's Social Systems Perspective (pp. 151-182). Copenhagen: Copenhagen Bussines School Press.

Corsi, G., Espósito, E. \& Baraldi, C. (1996). Glosario sobre la teoría social de Niklas Luhmann. México D.F.: Anthropos, Universidad Iberoamericana, ITESO.

CONACE (2003). Estrategia Nacional sobre Drogas 2003-2008. Santiago de Chile: Ministerio del Interior, Gobierno de Chile.

CONACE (2005). Intervención psicosocial. Prevención selectiva del consumo de drogas para niños/as y adolescentes en vulnerabilidad social. Santiago de Chile: Ministerio del Interior, Gobierno de Chile.

CONACE (2007). Escuela sin drogas. Santiago de Chile: Ministerio del Interior, Gobierno de Chile.

CONACE (2008). Consumo y tráfico de drogas en establecimientos educacionales. Guía de apoyo para Directores, Equipos Directivos y representantes de Consejos Escolares. Santiago de Chile: Ministerio del Interior, Gobierno de Chile. 
CONACE (2009). Modelo de intervención en sectores focalizados vulnerables al consumo y tráfico de drogas. Santiago de Chile: Ministerio del Interior, Gobierno de Chile.

CONACE (2011). Estrategia Nacional de Drogas y Alcohol 2011-2014. Santiago de Chile: Ministerio del Interior y Seguridad Pública. Gobierno de Chile.

CICAD (2010). Estrategia hemisférica de drogas, plan de acción 2011-2015. Organización de los Estados Americanos.

Gifre, M. \& Moisés, E. (2012). Consideraciones educativas de la perspectiva de Urie Bronfenbrenner. Contextos educativos, 15, 79-92.

Izuzquiza, I. (2008). La sociedad sin hombres. Niklas Luhmann o la teoría como escándalo. Barcelona: Anthropos.

Luhmann, N. (1993). Deconstruction as Second-Order Observing. New Literary History, 24(4), 763-782.

Luhmann, N. (1997). Observaciones de la modernidad. Racionalidad y contingencia en la sociedad moderna. Barcelona: Paidós.

Luhmann, N. (2007). La sociedad de la sociedad. México D.F.: Herder, Universidad Iberoamericana.

López, M. (2009). El concepto de anomia de Durkheim y las aportaciones teóricas posteriores. Iberofórum, 8, 130-147.

Montenegro, M. (2001). Conocimientos, agentes y articulaciones: Una perspectiva situada a la intervención social. Tesis doctoral, Universidad Autónoma de Barcelona.

Martínez, V. \& Covarrubias, G. (1999). La prevención de drogas en la comunidad escolar. Revista de psicología de la Universidad de Chile, 8(1), 131-143.

Martens, V. (2006). The Distinctions within Organizations: Luhmann from a Cultural Perspective. Organization, 13(1), 83-108.

Nassehi, A. (2005). Organizations as Decision Machines: Niklas Luhmann's Theory of Organized Social Systems. The Sociological Review, 53(1), 178-191.

Observatorio Chileno de Drogas (2006). Informe anual de la situación de drogas en Chile. Santiago de Chile: Ministerio del Interior, Gobierno de Chile.

OPECH (2005). SIMCE: Balance crítico y proyecciones imprescindibles. Documento de trabajo $n^{\circ} 1$. OPECH.

Pinilla, J. (2012). Think Tanks, saber experto y formación de agenda política en el Chile actual. Polis, 11(32), 119-140.

Redondo, J. (2005). El experimento chileno en educación: ¿Conduce a mayor equidad y calidad en la educación? Última Década, 22, 95-110.

Redondo, J. (2009). La educación chilena en una encrucijada histórica. Diversia, 1, 1339.

Rodríguez, D. (2004). Gestión organizacional. Elementos para su estudio. Santiago de Chile: Ediciones Universidad Católica de Chile.

Rodríguez, D. \& Arnold, M. (2007). Sociedad y teoría de sistemas. Santiago de Chile: Editorial Universitaria.

SENDA (2012). Noveno Estudio Nacional de Drogas en Población Escolar de Chile, 2011. Santiago de Chile: Ministerio del Interior y Seguridad Pública, Gobierno de Chile. 
SENDA (2012b). A Tiempo: Una estrategia de detección precoz y trabajo preventivo del consumo de drogas y alcohol en adolescentes. Santiago de Chile: Ministerio del Interior y Seguridad Pública, Gobierno de Chile.

Silva, J. (2009) Enseñanza municipal y el declive de la educación modernizadora. Diversia, 1, 41-69.

\section{FUENTES DE INTERNET}

SENDA (s.f. a) Presentación del programa Activa tu Desarrollo, Actitud. Ministerio del Interior y Seguridad Pública, Gobierno de Chile. Consultado en septiembre de 2013 desde: http://www.senda.gob.cl/prevencion/programas/escolar/actitud/\#.UddLtKy0SZk

SENDA (s.f. b) Actitud, ¡Activa tu desarrollo! Manuales del profesor de enseñanza básica. Ministerio del Interior y Seguridad Pública, Gobierno de Chile. Consultados en agosto de 2013 desde: http://www.senda.gob.cl/prevencion/programas/escolar/actitud/profesor1basico/ http://www.senda.gob.cl/prevencion/programas/escolar/actitud/profesor2basico/ http://www.senda.gob.cl/prevencion/programas/escolar/actitud/profesor3basico/ http://www.senda.gob.cl/prevencion/programas/escolar/actitud/profesor4basico/ http://www.senda.gob.cl/prevencion/programas/escolar/actitud/profesor5basico/ http://www.senda.gob.cl/prevencion/programas/escolar/actitud/profesor6basico/ http://www.senda.gob.cl/prevencion/programas/escolar/actitud/profesor7basico/ http://www.senda.gob.cl/prevencion/programas/escolar/actitud/profesor8basico/

SENDA (s.f. c) Actitud, ¡Activa tu desarrollo! Manuales del profesor de enseñanza media. Consultados en agosto de 2013 desde: http://www.senda.gob.cl/prevencion/programas/escolar/actitud/profesor1medio/ http://www.senda.gob.cl/prevencion/programas/escolar/actitud/profesor2medio/ http://www.senda.gob.cl/prevencion/programas/escolar/actitud/profesor3medio/ http://www.senda.gob.cl/prevencion/programas/escolar/actitud/profesor4medio/

UNICEF (1989) Convención sobre los Derechos del Niño. Organización de las Naciones Unidas. Consultado en junio de 2013 desde: http://www.unicef.cl/archivos documento/112/Convencion.pdf

\section{SOBRE EL AUTOR}

Patricio Espinosa Polanco es Profesional de apoyo en la Facultad de Ciencias Sociales, Universidad Católica de Temuco, Chile. Antropólogo por la Universidad Católica de Temuco. Entre sus áreas de especialización se encuentran: Teoría de Sistemas Sociales, Desarrollo y Territorio.

\section{CONTACTO}

Facultad de Ciencias Sociales, Universidad Católica de Temuco

Manuel Montt 056, Casilla 15 D

Código postal: 4813302

Temuco, Chile

patricioespinos@gmail.com 
Recibido: Septiembre 2013

Aceptado: Enero 2014 